\title{
Successfully Climbing the "STAIRs": Surmounting Failed Translation of Experimental Ischemic Stroke Treatments
}

\author{
Michael P. Kahle ${ }^{1,2}$ and Gregory J. Bix ${ }^{2,3}$ \\ ${ }^{1}$ Department of Neuroscience and Experimental Therapeutics, College of Medicine, Texas A\&M Health Science Center, \\ Bryan, TX 77807, USA \\ ${ }^{2}$ Sanders-Brown Center on Aging and Department of Anatomy and Neurobiology, University of Kentucky, \\ 430 Sanders Brown Building 800 S. Limestone Street, Lexington, KY 40536, USA \\ ${ }^{3}$ Department of Neurology, University of Kentucky, 430 Sanders Brown Building 800 S. Limestone Street, Lexington, \\ KY 40536-0230, USA
}

Correspondence should be addressed to Gregory J. Bix, gregorybix@uky.edu

Received 21 August 2012; Revised 7 November 2012; Accepted 16 December 2012

Academic Editor: Petra Henrich-Noack

Copyright ( $\odot 2012$ M. P. Kahle and G. J. Bix. This is an open access article distributed under the Creative Commons Attribution License, which permits unrestricted use, distribution, and reproduction in any medium, provided the original work is properly cited.

The Stroke Therapy Academic Industry Roundtable (STAIR) provided initial (in 1999) and updated (in 2009) recommendations with the goal of improving preclinical stroke therapy assessment and to increase the translational potential of experimental stroke treatments. It is important for preclinical stroke researchers to frequently consider and revisit these concepts, especially since promising experimental stroke treatments continue to fail in human clinical trials. Therefore, this paper will focus on considerations for several key aspects of preclinical stroke studies including the selection and execution of the animal stroke model, drug/experimental treatment administration, and outcome measures to improve experimental validity and translation potential. Specific points of interest discussed include the incorporation of human comorbid conditions and drugs, the benefits of defining a proposed mechanism of action, replication of results using multiple methods, using clinically relevant routes of administration and treatment time windows, and performing and reporting good experimental methods to reduce bias such as, as suggested by the updated STAIR recommendations, sample size calculations, randomization, allocation concealment, blinding, and appropriate inclusion/exclusion criteria. It is our hope that reviewing and revisiting these considerations will benefit researchers in their investigations of stroke therapies and increase the likelihood of translational success in the battle against stroke.

\section{Introduction}

Stroke is a leading cause of death and adult disability in the USA. A tremendous societal burden, stroke affects the lives of approximately 795,000 people each year, and costs reach 10 s of billions annually [1]. Unfortunately, however, despite decades of research, FDA approved care for ischemic stroke, the most common type of stroke that results from an obstruction (typically a blood clot) blocking a cerebral blood vessel, remains tissue plasminogen activator (tPA, originally approved in 1996) and endovascular clot retrieval/removal techniques including the MERCI device and PENUMBRA system (approved in 2004 and 2008, resp.) [2]. Furthermore, tPA administration must follow clinical validation that the stroke is ischemic and not hemorrhagic, is restricted to 3$4.5 \mathrm{hrs}$ after stroke onset, and risks hemorrhagic transformation, whereas devices require equipment and expertise [3]. Importantly, many stroke treatments have benefitted or seemingly cured stroke in animal models, but have ultimately failed in their translation to clinical trials. This has resulted in a waste of resources and loss of enthusiasm for novel approaches to stroke therapy and therefore necessitates discussion on how to surmount this tremendous barrier to science and medicine.

Increasing the quality of preclinical studies to overcome failed translation was the general goal of the Stroke Therapy Academic Industry Roundtable (STAIR) when it first gave "Recommendations for standards regarding preclinical 
neuroprotective and restorative drug development" in 1999 and updated recommendations in 2009 [4, 5]. Unfortunately, thirteen years since the original STAIR recommendations, we still face failure in developing new clinical stroke therapies. Although the STAIR recommendations are perhaps more widely known today, potential stroke therapies have not been subjected to more thorough preclinical evaluation. Furthermore, preclinical studies show huge variation in methodology. For example, in 54 studies examined, Philip et al. found 8 models of focal ischemia, 15 species, 6 histological stains for infarct size, and 19 behavioral tests in nonprimates [6]. Therefore, revisiting the STAIR recommendations to modify current practices/habits based on new considerations and knowledge may improve the odds of not being "lost in translation." To that end, we use the STAIRs as a platform to consider potential limitations of preclinical stroke investigations, as well as make suggestions as to how such limitations might be overcome.

\section{Animal Model}

Selecting the proper animal model amongst the large variety available to the preclinical stroke researcher should be done considering the following: (1) the species and strain, (2) the treatment's proposed mechanism of action, and (3) the incorporation of human comorbid conditions. The STAIR typically recommends using rats first, unless using genetically modified (e.g., transgenic, knockout) mice, and then replicating results in at least one other species, preferably gyrencephalic species and optimally nonhuman primates [4].

2.1. The Species and Strain. Importantly, one should not assume that inducing a similar surgical manipulation that is well characterized in one species (usually rat) will completely translate to another species at the cellular and molecular levels of pathophysiology. For example, the stereotactic injection of endothelin-1 to induce transient vasospasm and resultant occlusion of a cerebral blood vessel, routinely performed in rats, produces no infarct when administered alone in mice [7]. This principle also applies to differences between laboratory animal strains [8] and between genetically modified animals and wild types. Reasons for this discrepancy could include, for example, differences in neurovascular anatomy or collateral circulation that could affect the size of the tissue subjected to ischemia or the efficacy of compensatory mechanisms to reperfuse the ischemic area [9]. Indeed, in a global cerebral ischemia model, MF1 mice had less neuronal damage and ischemic stress, higher blood pressure, and greater Circle of Willis plasticity compared to the more commonly studied C57Bl/6J mice [10]. However, it has been argued that rodent ischemia consequences do closely replicate human pathobiology and that rodent models have similar vasculature to humans, whereas the largest disadvantages of rodent models are that they are not gyrencephalic, that white matter makes up a larger proportion in the human than the rodent brain, and that variability from rodent to rodent is actually too consistent to translate well to the heterogeneous human population that experiences stroke and is included in clinical trials (reviewed in Willing et al., 2009) [11]. Conversely, even within a single mouse strain, differences in the completeness of the Circle of Willis exist, leading some researchers to bypass this anatomic variability with a distal middle cerebral artery occlusion stroke model [12]. Finally, a practical consideration for model choice is how costly the experimental treatment is to the researcher. For example, a $1 \mathrm{mg} / \mathrm{kg}$ treatment dose would require more mass of drug to administer in a rat than in a mouse.

2.2. The Treatment's Proposed Mechanism of Action. As mechanisms of experimental stroke treatments may not translate to humans, it is logical to suggest that nonhuman primates, potentially the closest model to humans for stroke experimentation, should be used to perhaps minimize this problem. Indeed, similar stroke models (e.g., MCA occlusion models, autologous embolic models, photothrombosis, etc.) to rodents and behavioral tests to humans are available in lissencephalic and gyrencephalic nonhuman primates, where the Macaca fascicularis may be a good model given its gyrencephalic brain, relatively low level of collateralization, and ease of inducing stroke [13]. However, relatively few researchers today have the necessary experience, facilities, or access/approval to perform nonhuman primate work. Also, ethical considerations, expense, and subsequent difficulty of powering such studies with sufficient numbers of animals for valid statistical analysis are substantial. Fortunately, rodents are relatively affordable for experimental stroke study and can provide a source of genetic manipulation useful as proofof-concept for the treatment's mechanism of action. These genetic manipulations would perhaps ideally be performed in both positive and negative directions, for example, with transgenic animals that overexpress a component of the mechanism (positive animal manipulation) or hypomorph, knockdown, knockout, or dominant-negatives (negative animal manipulation). Additionally, more sophisticated and specific techniques could be performed including cellspecific or inducible genetic manipulations, for example, using the Tet-on system. Defining a therapy's proposed mechanism of action is important for clinical translation particularly if it is known that similar mechanisms might occur in humans, although this is not a guarantee for successful clinical translation. Furthermore, such knowledge could help predict potential deleterious treatment side effects; however, side effects (or lack thereof) in an animal model also may or may not translate to humans. Additionally, if a mechanism is defined, one could test another therapy that is more specific for that mechanism or (potentially easier for translation) test a currently FDA approved drug that is known to operate under the desired mechanism.

2.3. The Incorporation of Human Comorbid Conditions. This is likely the most important consideration in the selection of an animal model to increase the translation potential of preclinical stroke studies. Comorbid conditions for stroke primarily include older age, hypertension, diabetes, and 
hypercholesterolemia, emphasized in the updated STAIR recommendations as necessary to include in additional studies after studying young healthy males [5], as well as metabolic syndrome, heart disease (especially atrial fibrillation), smoking, and high alcohol consumption $[1,14]$. These conditions may strongly influence the pathophysiology and endogenous response to stroke. Therefore, comorbid conditions should be incorporated into preclinical studies and compounded with one another as much as possible to increase translation potential. For example, an 18-month aged mouse with hypertension and diabetes would more accurately represent the comorbidity often seen in human stroke patients than the 3-4 month healthy young adult mice typically used in preclinical studies. Additionally, studies should also incorporate more testing in females, as the susceptibility, processes and responses to stroke differ between genders $[15,16]$. In fact, women have a higher lifetime risk of stroke among those 55 to 75 years of age, $20-21 \%$ compared to $14-17 \%$ in men [1], and suffer a higher 30 -day mortality rate, $20 \%$ compared to $14 \%$ in men [17]; however, females remain underrepresented in experimental research.

Finally, potential stroke patients are more likely to be taking drugs to treat comorbid conditions, and these drugs could potentially interact directly or physiologically with experimental stroke treatments. For example, an individual with heart disease may be taking an ACE inhibitor, a $\beta$ blocker, and a statin, or an elderly woman may be taking calcium and vitamin D supplements for osteoporosis. The updated STAIR recommendations mention the need for such interaction studies with a therapeutic agent and medications commonly used in stroke patients [5]. Indeed, having these other bioactive compounds "on board" could profoundly impact how the brain suffers and responds to stroke, as well as the efficacy of a stroke treatment. A discussion of drugs that are administered at the time of stroke treatment is in the "Drug Administration: Amount and Polypharmacology" subsection. Therefore, incorporating "comorbid drugs" into preclinical studies could increase translation potential.

Collectively, to improve the likelihood of success in clinical trials, the animal model chosen in testing a preclinical stroke treatment should be appropriate for the stroke model used while eliminating potential confounding species/strain differences and by replication in a different species (as suggested in the STAIR recommendations) [4] to further define the therapy's mechanism of action via specific positive and negative genetically manipulated animals and to include comorbid conditions and drugs in humans.

\section{Stroke Model}

There are many ischemic stroke induction models, often with multiple variations and typically targeting the middle cerebral artery, available to the preclinical researcher including: endothelin-1 application, electrocoagulation, intraluminal filament (suture), clip or mechanical occlusion, photothrombosis, embolic models including administration of an autologous blood clot embolus, and thrombin injection. Each of these models has advantages and disadvantages, including perhaps the most clinically relevant model of autologous blood clot embolus that is associated with high variability in size and location as well as potential early autolysis, hemorrhage, and high mortality (reviewed by Macrae, 2011) [18]. Additionally, a model has recently been described using emboli made of "red" (erythrocyte/fibrin) clots, rather than traditional "white" (platelet/fibrin) clots, which may be more related to human stroke [19]. On the other hand, photothrombosis is comparatively very reproducible in infarct size and location, but is associated with increased edema not seen in human stroke [18]. Unfortunately, none of the models currently available can recapitulate all of the complicated facets of human stroke. Indeed, it has recently been proposed that the most commonly used type of stroke models, transient mechanical vascular occlusion (e.g., intraluminal filament model), should be eliminated entirely from preclinical stroke research. This argument was based on the position that transient mechanical vascular occlusion induces different pathophysiology and postischemic recirculation mechanisms allowing for a longer therapeutic window in experimental models than that available in human stroke $[20,21]$. However, although these models do not perfectly replicate human stroke, some considerations may increase translation potential including (1) the size of infarct generated, (2) the duration of ischemia, and (3) physiological monitoring.

3.1. The Size of Infarct Generated. Infarct size and location can vary greatly between and sometimes within stroke models, and global ischemia and models with very large infarcts may not translate well. Comparatively, distal middle cerebral artery occlusion and photothrombosis produce relatively small cortical infarcts, whereas the suture model can produce relatively large infarcts [18]. Large infarct models may be unfavorable as they often more accurately represent human malignant infarction than typical stroke [22]. This may also be a concern with genetically modified animals that have larger infarcts than wild types. Of particular concern, large infarcts can result in the destruction or the influence of tissue with regulatory roles (temperature, hormones, etc.), which may confound the investigation. For example, thalamic, hypothalamic, hippocampal, and midbrain damage can occur in intra-arterial suture occlusion (especially with occlusions greater than $60 \mathrm{~min}$ ), which is not seen in human stroke, and about $10 \%$ of mice develop subarachnoid hemorrhage [22]. Another consequence of very large infarcts is that animals may have a higher mortality rate, which would select only the population of surviving animals for outcome measures; however, properties that allowed that population to survive (other than the variable being tested) may confound the investigation and subsequent translation potential. Therefore, mortality numbers and causes should be reported. Finally, large infarcts may affect different neuronal types than those that are typically directly affected in human stroke. For example, if a treatment was more neuroprotective in hippocampal than cortical neurons, models that include hippocampal damage may show a decrease in infarct size with treatment, but if that reduction 
is due to the protection of hippocampal neurons not typically infarcted in human stroke, translation potential is confounded. Therefore, focal ischemia may be preferred over global ischemia and induction of smaller, more reproducible infarcts preferred over larger infarcts. However, available models that consistently produce small infarcts can also have limitations. For example, photothrombosis results in high vasogenic and cytotoxic edema more similar to traumatic brain injury than human stroke [22]. Additionally, reports of neuroprotection in very small infarcts may be misleading because a large percent change in infarct size may overestimate the benefit of a very small amount of salvaged tissue. Furthermore, distal MCA infarct models may produce small infarcts resulting in specific whisker barrel cortex deficits that are difficult to assess. This can be somewhat overcome by occluding the ipsilateral or both carotid arteries; however, the resultant increase in mortality has led to the development of a model that combines distal MCA occlusion with 1 hour of hypoxia, termed "DH stroke" [23]. DH stroke reportedly minimizes problems of Circle of Willis variability in $\mathrm{C} 57 \mathrm{BL} / 6 \mathrm{~J}$ mice, increases infarct size and behavioral effects, without impacting the hippocampus or significantly increasing mortality. Lastly, very small experimental infarcts could potentially underestimate the size of typical human ischemic stroke infarcts.

3.2. The Duration of Ischemia. How long a cerebral artery is occluded should also be taken into consideration and is reviewed in significant detail by Liu et al. [24]. In addition to increasing infarct size beyond what is seen in humans, prolonged occlusion may alter viable cell functioning of the neurovascular unit beyond human stroke, which may be difficult, if feasible, to detect. On the other hand, permanent occlusion models do not include a reperfusion injury component, which is likely relevant to stroke injury in humans, and collateral vessels may develop and restore blood flow to affected areas. Indeed, the rate of spontaneous recanalization/reperfusion is significant in humans and contributes to variability in pathophysiology and outcome [19]. Furthermore, as thrombolytics and clot retrieval and removal devices become even more effective, reperfusion and potential reperfusion injury may become more frequent. Therefore, to increase translation potential, an appropriate model should be chosen that represents the human condition with respect to the mechanisms of stroke being targeted and the treatment's proposed mechanism of action, especially when the therapy may be conducive to administration with tPA in humans.

3.3. Physiological Monitoring. Physiological parameters including blood gases, blood pressure, blood glucose level, body temperature, and cerebral blood flow (CBF) should be assessed during and beyond the stroke model procedure. For example, it is important to determine if an experimental treatment is solely affecting stroke infarct size or recovery via an indirect mechanism by altering physiological parameters (e.g., neuroprotective hypothermia). Therefore, due to inherent variability between and within models, researchers should also report in detail the inclusion/exclusion criteria used (including mortality), which may improve translation. Importantly, one factor that could influence physiological parameters is the anesthetic used, as some anesthetics are associated with neuroprotection, hypothermia, dehydration, or other effects [24-26]. Additionally, anesthetic effects may vary between species/strains, interact with the therapy, or influence the pathophysiology and response to stroke. For example, ketamine is classified as an NMDA receptor antagonist that has been found to interact at several other off-target sites as well $[27,28]$. Therefore, one could take the perspective that stroke studies testing a neuroprotective agent performed under anesthesia are actually testing a combinatorial treatment of two drugs [8]. Therefore, we suggest that experimental stroke results also be confirmed with an alternative anesthetic. CBF can be monitored using a laser Doppler system. Although the STAIR originally recommended decreasing laser Doppler signal by $\geq 60 \%$ to ensure appropriate ischemia [4], this seems too low and too broad a range for validity and consistency as the pathophysiology and responses to stroke could vary greatly between, for example, $61 \%$ and $97 \%$ occlusions. Instead, we suggest occlusions of $\geq 80-85 \%$ and for decreases in CBF to be compared between treatment groups to assess whether differences in CBF systematically correlate with outcome measures. Additionally, for transient models CBF should be monitored (1) before occlusion for a baseline reading, (2) at the start of occlusion to obtain a satisfactory reduction in $\mathrm{CBF},(3)$ at the end of occlusion to ensure that the vessel has remained occluded, and (4) at the beginning of reperfusion to verify that an increase in $\mathrm{CBF}$ from the occluded value is observed. Indeed, the STAIR update emphasized the importance of documenting adequate sustained occlusion and monitoring reperfusion by Doppler flow or perfusion imaging [5]. Despite this importance of CBF monitoring, Philip et al. reported that less than $30 \%$ of studies reported monitoring CBF [6].

Collectively, to increase the translational potential for human stroke therapy, the experimental stroke model should be performed with an appropriate model to produce infarcts that do not affect regulatory structures or induce side effects not seen in humans. Furthermore, physiological parameters should be monitored, especially CBF, to both validate the stroke procedure itself and determine whether the treatment has confounding effects on physiology.

\section{Drug Administration}

The (1) amount and polypharmacology, (2) timing, and (3) route of drug/treatment administration are extremely important not only for assessment of therapeutic efficacy in experimental models, but also for translational potential.

4.1. Amount and Polypharmacology. The STAIR recommendations place a strong emphasis on the need for researchers to define pharmacokinetics and pharmacodynamics including complete dose-response curves (preferably sigmoidalrather than bell—shaped) with additional minimum dose of 
effectiveness and maximum dose of tolerance from serum levels and consistent minimum neuroprotective concentration $[4,5]$. While these are extremely important for assessing translation potential, at the laboratory level these studies can be extremely time and resource costly and are likely not feasible to perform without first showing strong evidence of efficacy.

Another consideration is the use of the "cocktail approach" or "polypharmacology," also mentioned in the STAIR recommendations [4], where treatment involves modulation of multiple targets potentially with more than one drug (not necessarily at the same time). Stankowski and Gupta reviewed many of the early pathological mechanisms of stroke and emphasized the need for a neurotherapeutic that could interfere with several stroke processes at once [29]. Although the combinatorial drug approach is rarely used in laboratory stroke research, it has high potential and, therefore, should perhaps attract more attention. Important among combinatorial treatment testing will be novel treatments in conjunction with clinically relevant tPA (or another thrombolytic) $[30,31]$. Indeed, given the perspective that restoration of oxygen and glucose will always be the best neuroprotective therapy for ischemic stroke, and that tPA has many independent effects from thrombolysis (potentially due to its formulation with L-arginine), potential advantages and disadvantages of combinatorial therapy with tPA need to be assessed [32]. As discussed earlier, another aspect of the cocktail approach that should be considered is the use of a proposed treatment when drugs used for comorbid conditions are already "on board" to detect potential interactions that could be relevant in humans. Therefore, other drugs could be given before the onset of experimental stroke and still be "on board" following the stroke (modeling comorbid drugs, discussed above), or given at the time of stroke treatment (combinatorial, polypharmacology) in conjunction with the experimental treatment under study. Additionally, treatments that target different facets of stroke by different mechanisms, targets, and/or time frames could be combined for therapeutic benefit. For example, a drug to counteract excitotoxicity could be used acutely after stroke for the advantage of early neuroprotection but not more chronically where it might potentially impair neuroplasticity needed for neurorepair, combined with an antiinflammatory agent at a later or overlapping time frame, to avoid the limitation of inhibiting acute inflammatory responses that are necessary to rev up defenses, but for the advantage of reducing prolonged inflammation that may be detrimental to repair. These benefits may also be generally exploited by giving both a neuroprotective agent to reduce damage and a restorative agent to promote repair. In short, if the mechanisms of one drug are known, a second drug may be used to compliment the first drug, potentially additively or synergistically. However, with more drugs "on board" a major concern is the potential for neutralizing or harmful drug interactions and disruption of homeostatic balance, which would need to be investigated in detail [32]. Therefore, polypharmacology or the "cocktail approach" should be investigated more often but done so with caution as it may be a "high risk-high reward" approach to improve translation potential.

4.2. Timing. Stroke pathophysiology, endogenous responses, and opportunity for intervention are extremely time dependent, and researchers should attempt to use clinically relevant therapeutic windows in their preclinical studies of potential stroke treatments. This may limit the relevance of preconditioning and treatment concurrent with stroke onset studies to specific strokes that could be predicted to occur in a hospital/surgical environment, because we cannot typically predict when a stroke will occur or typically initiate immediate treatment. Indeed, the vast majority of stroke patients do not make the $3-4.5 \mathrm{~h}$ therapeutic window of $\mathrm{t}$ PA, thereby diminishing the clinical relevance of therapies administered within a couple of hours after stroke that might otherwise have been relevant in humans. Additionally, placing inpatients at risk of stroke on stroke "pretreatment" drugs may not be practical given their conditions. Furthermore, as stroke evolution and processes are not only prolonged but change with time, preconditioning and concurrent treatments may target pathology phases not targetable at feasible treatment windows (false positive for feasible therapeutic efficacy) and, conversely, may be limited in their ability to affect chronic injury mechanisms or repair (false negative for feasible therapeutic efficacy). Finally, an additional complication is the phenomenon of "wake-up" strokes, where the exact time of symptom onset may not be known. Although tPA has traditionally not been given to individuals who have suffered a wake-up stroke, recent evidence reported by Manawadu et al. at the American Heart Association International Stroke Conference (2012) suggests that $\mathrm{tPA}$ has similar functional and safety outcomes as compared to individuals treated $4.5 \mathrm{~h}$ after stroke onset [33]. Collectively, while stroke evolution varies between models, a potentially translatable stroke treatment should perhaps aim to have efficacy when administered at later time points, $\geq 3-$ 4.5 hours after injury in rodent models, and optimally with defined earliest and latest effective treatment times, thereby identifying relative rodent therapeutic windows as suggested by the STAIR recommendations [4].

4.3. The Route of Administration. Clinically relevant routes of administration, or effective routes of therapeutic administration that could safely/easily be used for a potential stroke patient, should be considered for preclinical stroke studies to increase translation potential. Although the route chosen will depend on properties of the current form of the treatment, and may be influenced by the integrity of the blood brain barrier at the time of treatment administration (although this barrier is significantly disrupted after stroke potentially minimizing this particular concern), researchers should consider that, for example, intraperitoneal (I.P.), intravenous (I.V.), intramuscular (I.M.), subcutaneous, or intranasal (among other potential administration routes) are more clinically relevant than intracerebroventricular (I.C.V.) or intrathecal routes, which are used to more directly target treatments to the site of stroke injury. As patients have 


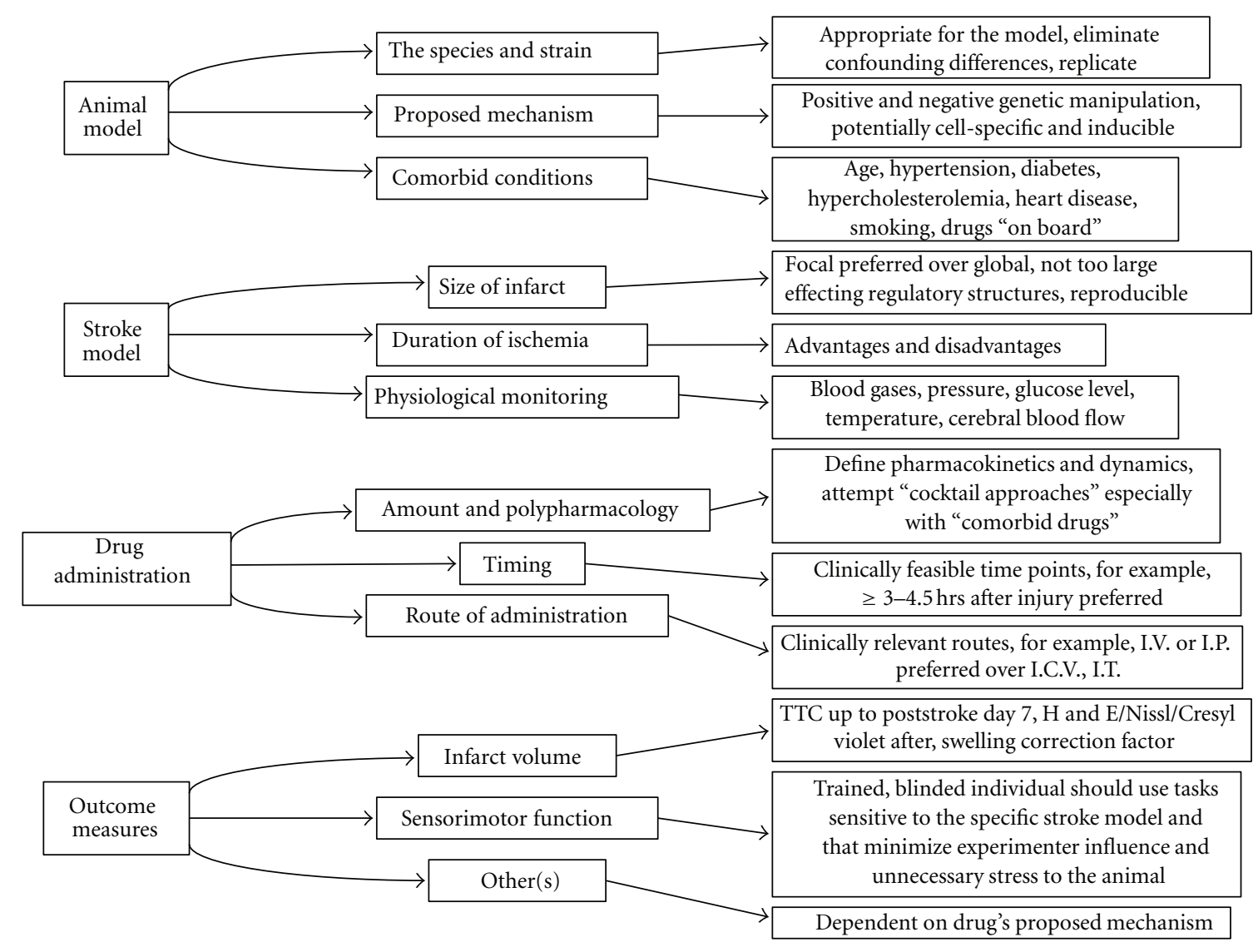

FIGURE 1: Summary of considerations for successfully climbing the STAIR.

I.V. access established in the hospital setting, and I.P., I.M., subcutaneous, or intranasal administration is relatively easy to accomplish, these methods are preferred over potentially damaging routes used in animals. Importantly, oral administration, although convenient, may be less clinically relevant if a patient is unconscious, has difficulty swallowing, or requires imminent surgery. Therefore, if an experimental stroke treatment could not be given via a clinically relevant route, researchers might consider additional or alternative methods. For example, reducing the chemical structure of the treatment to a smaller size that retains efficacy but achieves better distribution, using a similar treatment that operates via the same signal transduction mechanisms at the same level, or using a downstream effector of the treatment that operates via the same mechanism at a lower signal transduction level and can be given via a clinically relevant route.

Collectively, preclinical stroke studies of potential new treatments should be performed to identify the pharmacokinetics and pharmacodynamics of the treatment including dose response curves as much as practically possible, potentially with less emphasis on preconditioning or treatment administration at time of injury, with more efforts for "cocktail approaches" especially with "comorbid" drugs and with clinically relevant routes of administration.

\section{Outcome Measures}

The two most important and most commonly performed outcome measures, emphasized in the STAIR recommendations as both essential assessments [4], are measurement of infarct volume and sensorimotor function tests. These are both critical to perform in a valid manner to increase translation potential of preclinical stroke studies. Additionally, it is essential that the individual performing the outcome measures is blind to the treatment of each animal, and testing should be randomized. Indeed, the STAIR recommends reporting sample size calculations, proper randomization, and allocation concealment; however, Philip et al. reported that only $10 \%$ of studies reported whether the investigator was blinded during treatment administration $[5,6]$. Likewise, a systematic analysis of all studies published in The Journal of Cerebral Blood Flow and Metabolism in 2008 recently showed that of animal studies, only $22 \%$ reported randomization, $8 \%$ allocation concealment, $15 \%$ blinded assessment of outcome, and 14\% inclusion/exclusion criteria [34]. It will be critical to improve upon these measures and report them to ensure research validity.

5.1. Measurement of Infarct Volume. The most common measurements of preclinical experimental infarct volume 
are determined from brain tissue histologically stained with triphenyl tetrazolium chloride (TTC), hematoxylin and eosin (H\&E), nissl, or cresyl violet. TTC staining may be accurately used through poststroke day 7 [35], but is unreliable at later time points as infarct infiltration of inflammatory cells may confound measurement (by making the infarct artificially appear smaller). Furthermore, when quantifying infarct volume, a correction factor should be applied based on the swelling of the ipsilateral (stroked) hemisphere and apparent infarct so that infarct size quantification is not confounded by volumetric changes due only to swelling, of which edema is a major factor.

5.2. Sensorimotor Function Tests. The appropriate choice of functional tests in preclinical studies is also essential for increasing the translational potential of a stroke treatment. Common functional tests include the cylinder test, pawplacement reflex tests, ladder tests, beam tests, the gridwalking test, reaching tasks, and the rotarod task, among others. Each of these tests has advantages and disadvantages and which to choose will ultimately depend on the stroke model used (e.g., a stroke model that results in paw motor deficits should be followed by tests that involve paw movement, etc.) and resultant dysfunction displayed. General recommendations include randomized testing by the same tester who is trained to perform the tests validly and consistently, who is blinded to the treatment condition of animals (to optimize reproducibility and minimize bias), and reducing tester subjectivity by using more simple and natural tasks that minimize anxiety, fear, or hyperactivity of the animal. For example, a test in which a reflex-paw placement is elicited by touching the ipsilateral vibrissae on a hard surface may be complicated in mice due to the animal's resistance to being positioned in such a way, resulting in variability in animal mobility, temperament, motivation, and so forth, collectively resulting in unreliable measurements. Also, some reaching tasks involving food deprivation may introduce confounds associated with being food deprived, including hormone and motivation changes, in addition to potential confounding hunger changes from the drug. Thus, in agreement with the STAIR, researchers should perform more than one behavioral test that is distinct, sensitive to the deficit, and minimizes confounding influence on the animal.

The duration of sensorimotor testing is also an important consideration to ensure stable outcomes. Although the original STAIR recommendations mention that many preclinical studies only assess outcomes 1 day after ischemia, it appears that researchers now typically assess behavior for longer time periods after stroke onset. Prolonged testing is important as at 1 day after ischemia the infarct size has often not fully evolved in many stroke models, and outcomes are not stable. Additionally, some therapies may have early poststroke benefits that are later reversed or do not affect later phase detrimental mechanisms. Therefore, we recommend that when possible, functional testing should be carried out for at least 2 to 3 weeks after injury and longer if one or more groups do not have stable function. Finally, experimental animals possess some spontaneous recovery from strokeinduced sensorimotor dysfunction that can be quite robust; therefore, the effect of the treatment must be verified as distinct from spontaneous recovery.

The selection and necessity of other outcome measures depends on the treatment's proposed mechanism of action. For example, a proposed antiinflammatory mechanism might have outcome measures assessing inflammatory cells, markers, signals, and so forth. whereas an anti-apoptotic agent might perform TUNEL, caspase-3 cleavage, AnnexinV stains, and so forth. Additionally, these endpoints may be of high clinical relevance if translated as relevant biomarkers that could be measured directly or indirectly in humans, as suggested in the updated STAIR recommendations [5].

Collectively, outcome measures should include blinded volumetric measurement of the infarct area using the appropriate stain with correction for swelling, and at least two distinct sensorimotor function tests selected for sensitivity to the specific deficits, conducted in a manner to minimize tester bias and confounding influence on the animal, and performed for a prolonged duration to ensure outcome stability.

\section{Conclusions}

To increase the potential of laboratory experimental stroke treatments to translate to successful clinical trials, researchers have many factors to consider. These considerations, summarized in Figure 1, should include the animal model, stroke model, drug administration, and outcome measures to ensure that each aspect is as clinically relevant as practically possible. In general, replication of findings in multiple stroke models by multiple independent investigators is strongly encouraged, as suggested in the updated STAIR recommendations. While these considerations and STAIR may seem steep, the end results of potential translational success are worth the climb.

\section{Acknowledgment}

The authors would like to thank Dr. Farida Sohrabji for very useful conversations on the subject.

\section{References}

[1] V. L. Roger, A. S. Go, D. M. Lloyd-Jones et al., "Heart disease and stroke statistics-2012 update: a report from the american heart association," Circulation, vol. 125, no. 1, pp. e2-e220, 2012.

[2] N. Chaudhary, A. S. Pandey, and J. J. Gemmete, "Intervention in acute cerebral ischaemic stroke: a review of the role of pharmacological therapies and intra-arterial mechanical thrombectomy devices," Postgraduate Medical Journal, vol. 87, no. 1032, pp. 714-723, 2011.

[3] S. J. Maiser, A. L. Georgiadis, M. F. K. Suri, G. Vazquez, K. Lakshminarayan, and A. I. Qureshi, "Intravenous recombinant tissue plasminogen activator administered after $3 \mathrm{~h}$ following onset of ischaemic stroke: a metaanalysis," International Journal of Stroke, vol. 6, no. 1, pp. 25-32, 2011. 
[4] M. Fisher, "Recommendations for standards regarding preclinical neuroprotective and restorative drug development," Stroke, vol. 30, no. 12, pp. 2752-2758, 1999.

[5] M. Fisher, G. Feuerstein, D. W. Howells et al., "Update of the stroke therapy academic industry roundtable preclinical recommendations," Stroke, vol. 40, no. 6, pp. 2244-2250, 2009.

[6] M. Philip, M. Benatar, M. Fisher, and S. I. Savitz, "Methodological quality of animal studies of neuroprotective agents currently in phase II/III acute ischemic stroke trials," Stroke, vol. 40, no. 2, pp. 577-581, 2009.

[7] N. Horie, A. L. Maag, S. A. Hamilton, H. Shichinohe, T. M. Bliss, and G. K. Steinberg, "Mouse model of focal cerebral ischemia using endothelin-1," Journal of Neuroscience Methods, vol. 173, no. 2, pp. 286-290, 2008.

[8] L. Belayev, "Overcoming barriers to translation from experimental stroke models," in Translational Stroke Research, P. A. Lapchak and J. H. Zhang, Eds., vol. 3, chapter 24, pp. 471-492, Springer, 2012.

[9] K. Maeda, R. Hata, and K. A. Hossmann, "Differences in the cerebrovascular anatomy of C57Black/6 and SV129 mice," NeuroReport, vol. 9, no. 7, pp. 1317-1319, 1998.

[10] S. Kelly, J. McCulloch, and K. Horsburgh, "Minimal ischaemic neuronal damage and HSP70 expression in MF1 strain mice following bilateral common carotid artery occlusion," Brain Research, vol. 914, no. 1-2, pp. 185-195, 2001.

[11] A. E. Willing, "Experimental models: help or hindrance," Stroke, vol. 40, no. 3, pp. S152-S154, 2009.

[12] B. W. McColl, H. V. Carswell, J. McCulloch, and K. Horsburgh, "Extension of cerebral hypoperfusion and ischaemic pathology beyond MCA territory after intraluminal filament occlusion in C57Bl/6J mice," Brain Research, vol. 997, no. 1, pp. 15-23, 2004.

[13] D. J. Cook and M. Tymianski, "Nonhuman primate models of stroke for translational neuroprotection research," Neurotherapeutics, vol. 9, no. 2, pp. 371-379, 2012.

[14] S. Ankolekar, S. Rewell, D. W. Howells, and P. M. Bath, "The influence of stroke risk factors and comorbidities on assessment of stroke therapies in humans and animals," International Journal of Stroke, vol. 7, no. 5, pp. 386-397, 2012.

[15] M. Wiszniewska, M. Niewada, and A. Czlonkowska, "Sex differences in risk factor distribution, severity, and outcome of ischemic stroke," Acta Clinica Croatica, vol. 50, no. 1, pp. 21-28, 2011.

[16] M. Liu, S. Dziennis, P. D. Hurn, and N. J. Alkayed, "Mechanisms of gender-linked ischemic brain injury," Restorative Neurology and Neuroscience, vol. 27, no. 3, pp. 163-179, 2009.

[17] V. L. Roger, A. S. Go, D. M. Lloyd-Jones et al., "Heart disease and stroke statistics-2011 update: a report from the American Heart Association," Circulation, vol. 123, no. 4, pp. e18-e209, 2011.

[18] I. M. Macrae, "Preclinical stroke research—advantages and disadvantages of the most common rodent models of focal ischaemia," British Journal of Pharmacology, vol. 164, no. 4, pp. 1062-1078, 2011.

[19] K. Guluma, "Clinical relevance in a translational rodent model of acute ischemic stroke: incorporating the biological variability of spontaneous recanalization," in Translational Stroke Research, P. A. Lapchak and J. H. Zhang, Eds., vol. 3, chapter 26, pp. 525-540, Springer, 2012.

[20] K. A. Hossmann, "Cerebral ischemia: models, methods and outcomes," Neuropharmacology, vol. 55, no. 3, pp. 257-270, 2008.
[21] K. A. Hossmann, "The two pathophysiologies of focal brain ischemia: implications for translational stroke research," Journal of Cerebral Blood Flow and Metabolism, vol. 32, no. 7, pp. 1310-1316, 2012.

[22] S. T. Carmichael, "Rodent models of focal stroke: size, mechanism, and purpose," NeuroRx, vol. 2, no. 3, pp. 396-409, 2005.

[23] K. P. Doyle, N. Fathali, M. R. Siddiqui, and M. S. Buckwalter, "Distal hypoxic stroke: a new mouse model of stroke with high throughput, low variability and a quantifiable functional deficit," Journal of Neuroscience Methods, vol. 207, no. 1, pp. 31-40, 2012.

[24] S. Liu, G. Zhen, B. P. Meloni, K. Campbell, and H. R. Winn, "Rodent stroke model guidelines for preclinical stroke trials (1st Edition)," Journal of Experimental Stroke \& Translational Medicine, vol. 2, no. 2, pp. 2-27, 2009.

[25] H. Sakai, H. Sheng, R. B. Yates, K. Ishida, R. D. Pearlstein, and D. S. Warner, "Isoflurane provides long-term protection against focal cerebral ischemia in the rat," Anesthesiology, vol. 106, no. 1, pp. 92-99, 2007.

[26] L. C. Mei, J. Yang, S. Kem, L. Klaidman, T. Sugawara, and P. H. Chan, "Nicotinamide and ketamine reduce infarct volume and DNA fragmentation in rats after brain ischemia and reperfusion," Neuroscience Letters, vol. 322, no. 3, pp. 137-140, 2002.

[27] Y. L. Zhang, P. B. Zhang, S. D. Qiu, Y. Liu, Y. F. Tian, and Y. Wang, "Effects of ketamine-midazolam anesthesia on the expression of NMDA and AMPA receptor subunit in the periinfarction of rat brain," Chinese Medical Journal, vol. 119, no. 18, pp. 1555-1562, 2006.

[28] M. Proescholdt, A. Heimann, and O. Kempski, "Neuroprotection of $\mathrm{S}(+)$ ketamine isomer in global forebrain ischemia," Brain Research, vol. 904, no. 2, pp. 245-251, 2001.

[29] J. N. Stankowski and R. Gupta, "Therapeutic targets for neuroprotection in acute ischemic stroke: lost in translation?" Antioxidants and Redox Signaling, vol. 14, no. 10, pp. 1841$1851,2011$.

[30] R. J. Turner, G. C. Jickling, and F. R. Sharp, “Are underlying assumptions of current animal models of human stroke correct: from STAIRs to high hurdles?" Translational Stroke Research, vol. 2, no. 2, pp. 138-143, 2011.

[31] A. B. Singhal, E. H. Lo, T. Dalkara, and M. Moskowitz, "Ischemic stroke: basic pathophysiology and neuroprotective strategies," in Acute Ischemic Stroke, R. G. Gonzalez et al., Ed., chapter 1, pp. 1-24, Springer, Berlin, Germany, 2011.

[32] B. A. Sutherland, J. Minnerup, J. S. Balami et al., "Neuroprotection for ischaemic stroke: translation from the bench to the bedside," International Journal of Stroke, vol. 7, no. 5, pp. 407418, 2012.

[33] B. S. D. Manawadu, J. Jarosz, and L. Kalra, "Abstract 56: thrombolysis in selected patients with wake up stroke is feasible with similar safety as thrombolysis in 0-4.5 hours," Stroke, vol. 43, Article ID A56, 2012.

[34] H. V. Vesterinen, K. Egan, A. Deister, P. Schlattmann, M. R. MacLeod, and U. Dirnagl, "Systematic survey of the design, statistical analysis, and reporting of studies," Journal of Cerebral Blood Flow and Metabolism, vol. 31, no. 4, pp. 10641072, 2011.

[35] F. Liu, D. P. Schafer, and L. D. McCullough, "TTC, Fluoro-Jade $B$ and NeuN staining confirm evolving phases of infarction induced by middle cerebral artery occlusion," Journal of Neuroscience Methods, vol. 179, no. 1, pp. 1-8, 2009. 


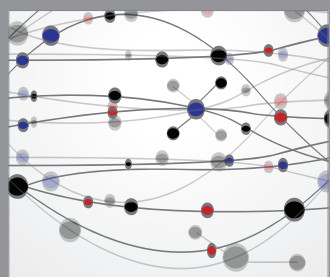

The Scientific World Journal
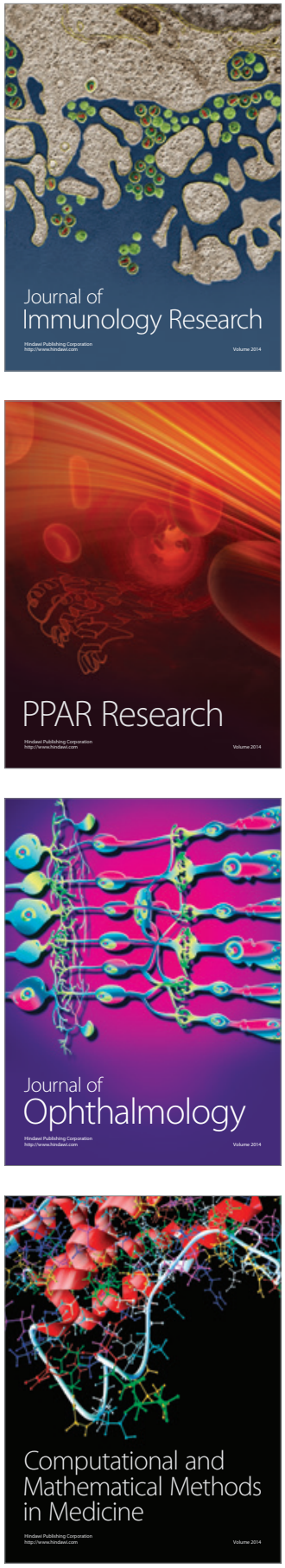

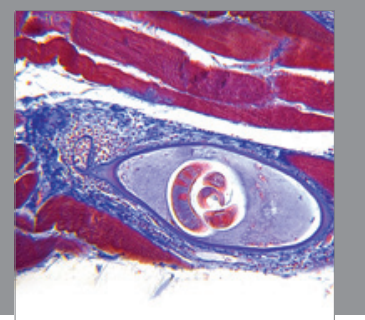

Gastroenterology

Research and Practice
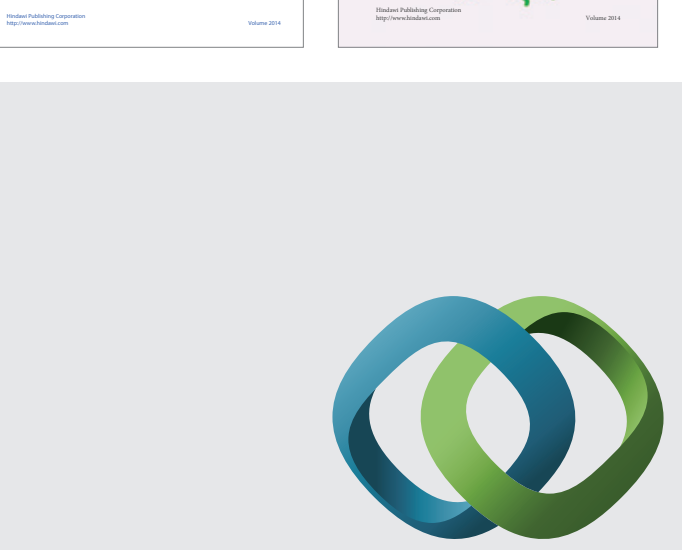

\section{Hindawi}

Submit your manuscripts at

http://www.hindawi.com
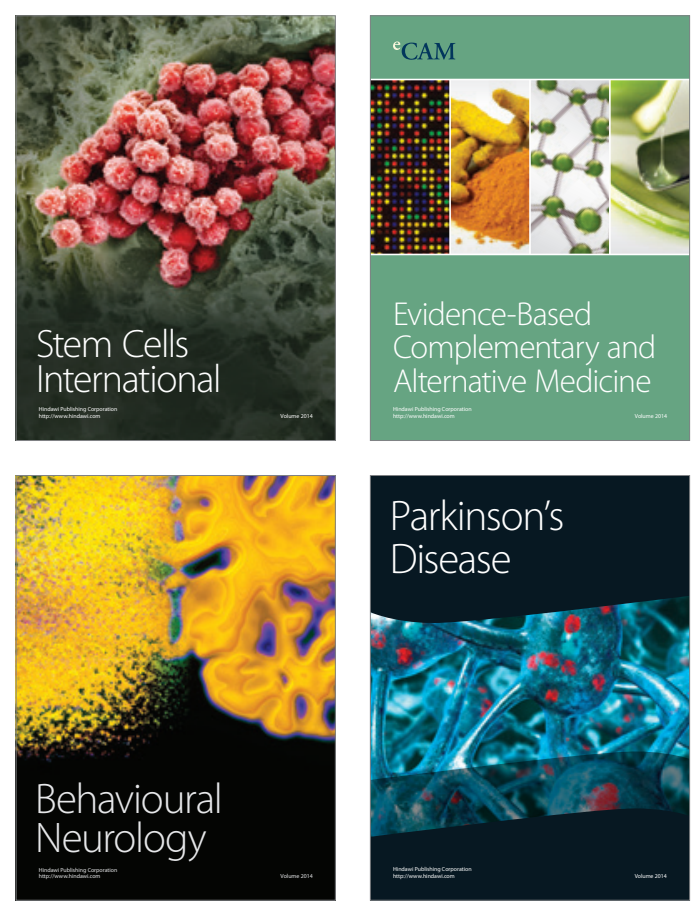

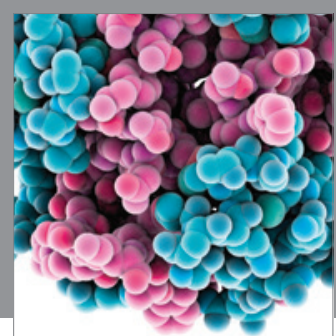

Journal of
Diabetes Research

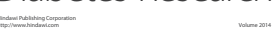

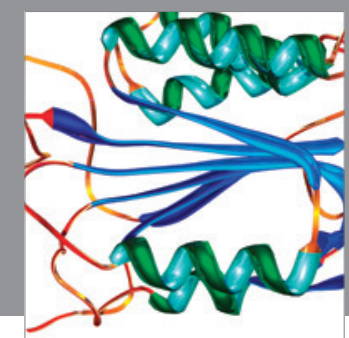

Disease Markers
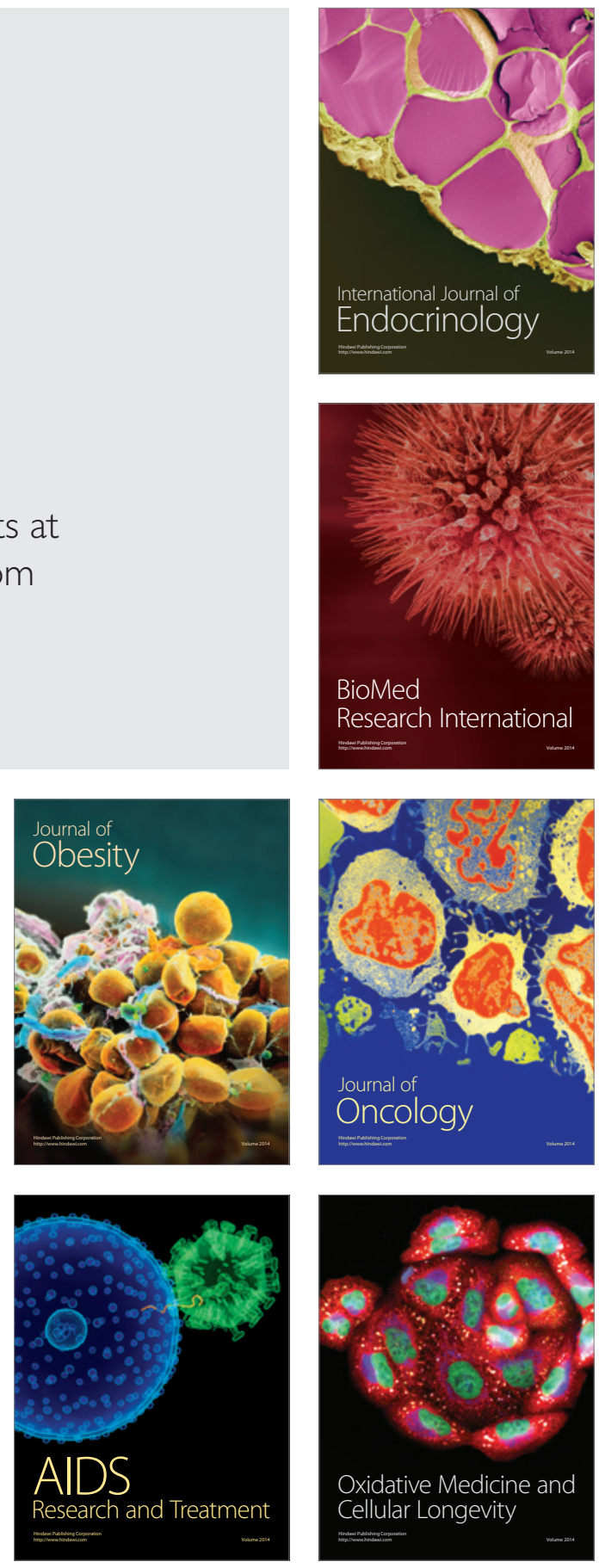